\title{
CK2 inhibition with silmitasertib promotes methuosis-like cell death associated to catastrophic massive vacuolization of colorectal cancer cells
}

\author{
Eduardo Silva-Pavez ${ }^{1}$, Paulina Villar ${ }^{1}$, César Trigo ${ }^{1}$, Esteban Caamaño ${ }^{1}$, Ignacio Niechi ${ }^{1,6}$, Pablo Pérez ${ }^{1}$, Juan P. Muñoz ${ }^{1}$, \\ Francisco Aguayo ${ }^{1}$, Verónica A. Burzio ${ }^{2}$, Manuel Varas-Godoy $\mathbb{B}^{3}$, Ariel F. Castro ${ }^{4}$, María I. Colombo ${ }^{5}$ and Julio C. Tapia ${ }^{1}$
}

\begin{abstract}
Protein kinase CK2 is a highly conserved and constitutively active Ser/Thr-kinase that phosphorylates a large number of substrates, resulting in increased cell proliferation and survival. A known target of CK2 is Akt, a player in the PI3K/Akt/ mTORC1 signaling pathway, which is aberrantly activated in 32\% of colorectal cancer (CRC) patients. On the other hand, mTORC1 plays an important role in the regulation of protein synthesis, cell growth, and autophagy. Some studies suggest that CK2 regulates mTORC1 in several cancers. The most recently developed CK2 inhibitor, silmitasertib (formerly CX-4945), has been tested in phase I/II trials for cholangiocarcinoma and multiple myeloma. This drug has been shown to induce autophagy and enhance apoptosis in pancreatic cancer cells and to promote apoptosis in nonsmall cell lung cancer cells. Nevertheless, it has not been tested in studies for CRC patients. We show in this work that inhibition of CK2 with silmitasertib decreases in vitro tumorigenesis of CRC cells in response to G2/M arrest, which correlates with $\mathrm{mTORC} 1$ inhibition and formation of large cytoplasmic vacuoles. Notably, molecular markers indicate that these vacuoles derive from massive macropinocytosis. Altogether, these findings suggest that an aberrantly elevated expression/activity of CK2 may play a key role in CRC, promoting cell viability and proliferation in untreated cells, however, its inhibition with silmitasertib promotes methuosis-like cell death associated to massive catastrophic vacuolization, accounting for decreased tumorigenicity at later times. These characteristics of silmitasertib support a potential therapeutic use in CRC patients and probably other CK2-dependent cancers.
\end{abstract}

\section{Introduction}

Colorectal cancer (CRC) is a multifactorial disease affecting millions of people worldwide and has been linked to deregulation of several signaling pathways. The PI3K/Akt signaling pathway plays an important role in a variety of cancers due to its association with processes that promote proliferation, resistance to apoptosis,

\footnotetext{
Correspondence: Julio C. Tapia (jtapia@med.uchile.cl)

'Departamento de Oncología Básico Clínica, Facultad de Medicina,

Universidad de Chile, Santiago, Chile

${ }^{2}$ Fundación Ciencia \& Vida, Andes Biotechnologies SpA, Facultad de Ciencias de la Vida, Universidad Andrés Bello, Santiago, Chile

Full list of author information is available at the end of the article.

Edited by A. Oberst
}

invasion, and metastasis ${ }^{1}$. In CRC, a number of genetic and epigenetic alterations have been described, for example, activating mutations in the PI3K kinase gene have been identified in $32 \%$ of tumors ${ }^{2}$, as well as loss of function mutations of the tumor suppressor $\mathrm{PTEN}^{3}$. All these alterations contribute to the aberrant activation of the PI3K/Akt signaling pathway and, in consequence, acquisition of a metastatic phenotype ${ }^{4}$.

A key downstream component of the PI3K/Akt signaling pathway is the mammalian target of rapamycin complex 1 (mTORC1), which plays an important role in different types of cancer, including $\mathrm{CRC}^{4,5}$. The core component of this complex, the mammalian target of

\section{(c) The Author(s) 2019}

(c) (i) Open Access This article is licensed under a Creative Commons Attribution 4.0 International License, which permits use, sharing, adaptation, distribution and reproduction c. in any medium or format, as long as you give appropriate credit to the original author(s) and the source, provide a link to the Creative Commons license, and indicate if changes were made. The images or other third party material in this article are included in the article's Creative Commons license, unless indicated otherwise in a credit line to the material. If material is not included in the article's Creative Commons license and your intended use is not permitted by statutory regulation or exceeds the permitted use, you will need to obtain permission directly from the copyright holder. To view a copy of this license, visit http://creativecommons.org/licenses/by/4.0/. 
rapamycin (mTOR), is a highly conserved Ser/Thr-kinase that integrates growth factor and nutritional signals to promote growth and survival of normal cells. Activation of mTORC1 leads to phosphorylation of mediators of protein translation and cell growth, including the ribosomal S6 kinase 1 (S6K1) and 4EBP1 ${ }^{6,7}$. MTORC1 plays an important role in the regulation of protein synthesis, cell growth and autophagy in response to nutrients and growth factors ${ }^{8}$. Inactivation of TSC2 by Akt favors the activation of Rheb, which interacts and activates mTORC1 at the lysosomal membrane ${ }^{8,9}$. Inhibition mTORC1 was shown to decrease formation of polyps, oncogenesis, and mortality of Apc $\Delta 716$ mice $^{10}$. Also, treatment with rapamycin leads to a reduction of tumors in an in vivo model of PI3K-dependent $\mathrm{CRC}^{11}$.

Autophagy is initiated by ULK-1, which is activated under nutrient deprivation or mTORC1 inhibition by rapamycin ${ }^{12-14}$. Autophagy is associated to a number of diseases, although its role in tumorigenesis and progression is controversial ${ }^{12,15}$. Some studies show that autophagy suppresses tumorigenesis ${ }^{15,16}$, while in others autophagy inhibition by silencing Rheb decreases survival of Colo320HSR colon cancer cells ${ }^{17}$. Likewise, autophagy inhibition exerts an anticancer effect in HCT-116 colon cancer cells by triggering apoptosis ${ }^{18}$. Conversely, a dual inhibitor of mTORC1/2, WYE354, induces autophagy and activates apoptosis in HCT-116 and HT-29 colon cancer cells ${ }^{19}$. Finally, Beclin-1 overexpression correlates with a positive prognosis and survival of CRC patients ${ }^{20}$.

Protein kinase CK2 has been proposed as a therapeutic target in various cancers. CK2 is a highly conserved constitutively active Ser/Thr-kinase capable of phosphorylating a large number of substrates, increasing proliferation, and survival ${ }^{21-23}$. CK2 is able to control mTORC1 in several cancers. In fact, CK2 regulates the PI3K/Akt pathway through phosphorylation of Akt at Ser129 , causing its hyperactivation ${ }^{24,25}$. Thus, CK2 silencing has been tested and greater effort dedicated to study specific inhibitors for therapy. The latest developed CK2 inhibitor, silmitasertib (formerly CX-4945), displays excellent pharmacological properties, which rendered it suitable for evaluation in phase I/II trials for cholangiocarcinoma and multiple myeloma (clinicaltrials.gov). Despite it has not yet been included in studies for CRC patients, it induces in vitro autophagy and enhances apoptosis in pancreatic cancer cells ${ }^{26}$, as well as promotes apoptosis in non-small cell lung cancer cells by inhibiting the PI3K/Akt/mTOR pathway ${ }^{27}$. In addition, silmitasertib induces apoptosis in epidermoid carcinoma and squamous carcinoma cells by a complete inhibition of the PI3K/Akt/mTOR pathway in combination with erlotinib $^{28}$.

Here, we show that an aberrantly elevated expression/ activity of CK2 may play an undescribed role in viability of
CRC cells. Thus, CK2 inhibition with silmitasertib promotes an early methuosis-like cell death, which is associated to massive catastrophic macropinocytosis, accounting for abolition of tumorigenicity at later stages. These results support the use of silmitasertib as a promissory therapeutic alternative in the treatment of CRC patients.

\section{Results \\ CK2 inhibition decreases tumorigenesis of CRC cells through a G2/M arrest}

Tumorigenic cells have the in vitro capacity of anchorage-independent growth and forming colonies in soft-agar. Thus, we studied whether CK2 inhibition could decrease clonogenesis of DLD-1 cells after 21 days of treatment with increasing concentrations of silmitasertib. A significant dose-dependent decrease in both number and size of the colonies was observed (Fig. 1), suggesting a potent effect of silmitasertib in decreasing CRC tumorigenesis. To elucidate the underlying mechanism, a tenfold shorter time was used to evaluate whether silmitasertib affects the viability of DLD-1 and other CRC cells of different malignancy ${ }^{29}$. SW-480 (Dukes B state), DLD-1 (Dukes C state), HT-29 (Dukes C/D state), and HCT-116 (Dukes D state) cells were grown with or without $25 \mu \mathrm{M}$ silmitasertib for $48 \mathrm{~h}$. Viability of all CRC cells decreased $60-70 \%$, while normal colon epithelial CoN cells only decreased just over 30\% (Fig. 2a). Decreased viability of CRC cells correlated with diminished proliferation (Fig. 2b) and late apoptosis, as observed by PARP-1 cleavage and annexin- $\mathrm{V}^{+} / \mathrm{PI}^{+}$ staining after $48 \mathrm{~h}$ (Fig. 2c, d), however, the pan-caspase inhibitor Z-VAD-FMK was unable to revert the decreased viability both at $24 \mathrm{~h}$ and $48 \mathrm{~h}$ (Supp Fig. 1A). Since double annexin $-\mathrm{V}^{+} / \mathrm{PI}^{+}$staining is also related to early necrosis/necroptosis, we assessed whether the decreased viability may probably derive from this at $48 \mathrm{~h}$. The necroptosis inhibitor, necrostatin-1s (Nec1s), did not revert the viability neither at $24 \mathrm{~h}$ nor at $48 \mathrm{~h}$ (Supp Fig. 1B). Additionally, localization of High Mobility Group Box 1 (HMGB1) transcription factor, which is exported to the cytoplasm during early necrosis ${ }^{30}$, showed only a minimal cytoplasmic localization at $12 \mathrm{~h}$ of treatment (Supp Fig. 1C).

A more specific analysis of silmitasertib treatment on cell cycle was performed. This analysis showed a basal sub- $\mathrm{G}_{0}$ fraction at $16 \mathrm{~h}$ (Fig. 3a) that was maintained at 24 h (Fig. 3b) but, as expected, significantly progressed at 48 h (Fig. 3c). In parallel, a reduction in the $G_{0} / G_{1}$ fraction was already observed at $16 \mathrm{~h}$, which progressed through $24 \mathrm{~h}$ and further at $48 \mathrm{~h}$. Notably, an increase in G2/M arrest was observed at $24 \mathrm{~h}$, which was much more elevated at $48 \mathrm{~h}$ of treatment. Activating mutation of the PIK3CA gene is a common trait in CRC tumors, leading 


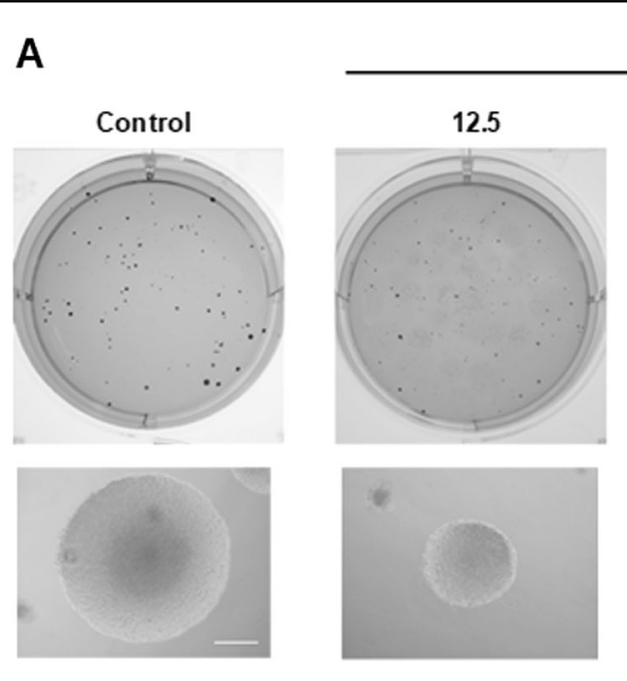

Silmitas ertib $(\mu \mathrm{M})$

B
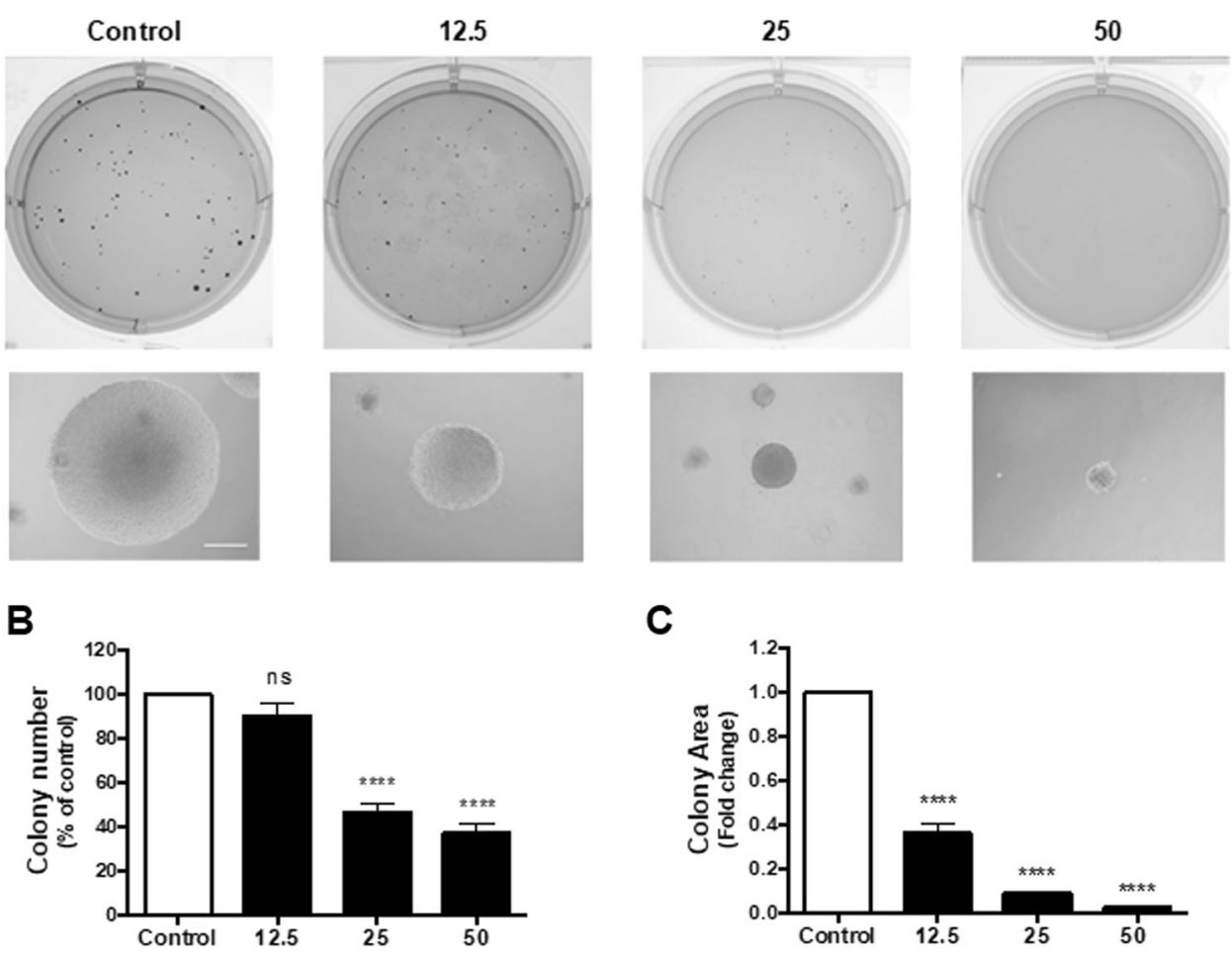

C

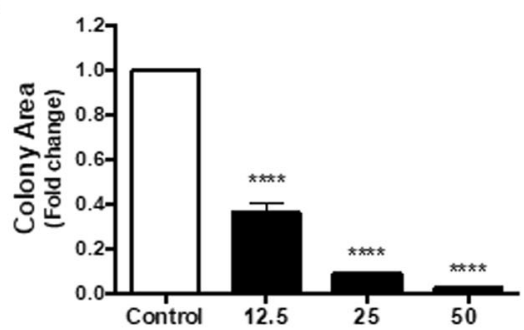

Fig. 1 CK2 inhibition with silmitasertib decreases in vitro tumorigenesis of CRC cells. Anchorage-independent colony growth in soft-agar was determined in DLD-1 cells treated with increasing concentrations of silmitasertib for 21 days. a Representative images of colonies in soft-agar of control (white bars) and silmitasertib-treated (black bars) cells. The number of colonies per well (b) and area of colonies (c) was plotted. Data represents the average $\pm \operatorname{SEM}(n=3) ;{ }^{* * *} p<0.0001 ; n s$, not significant. Bar: $20 \mu \mathrm{m}$

to aberrant activation of the PI3K/Akt pathway ${ }^{2}$, however, silmitasertib triggered the same G2/M arrest in SW-480 cells (Supp Fig. 2) which, unlike DLD-1, does not contain activating mutations in PIK3CA. These results suggest an effect of the CK2 inhibitor silmitasertib on decreasing tumorigenicity of CRC cells through a G2/M arrest.

\section{CK2 regulates mTORC1 activity in CRC cells}

Since mTORC1 is activated during mitosis and is therefore important in $\mathrm{G} 2 / \mathrm{M}$ cell cycle progression, as well as in maintaining genomic integrity during this per$\operatorname{iod}^{27,31-34}$, we assessed whether the G2/M arrest could be a consequence of reduced mTOR activity. Thus, phosphorylations on S6K1 at Thr389 and S6 at Ser235/Ser236 were used as activity markers of kinases mTORC1 and S6K1, respectively. As observed in Fig. 4a, treatment with silmitasertib for $16 \mathrm{~h}$ led to reduced activity of both kinases. To confirm that the effect on mTORC1 is specific of CK2, expression of CK2 $\alpha$ was decreased with a specific siRNA $^{35}$ and levels of pT389-S6K1 were analyzed. Concordantly, a similar decrease in pT389-S6K1 levels was observed as early as $16 \mathrm{~h}$ upon silencing of CK2 $\alpha$ (Fig. 4b). Given that mTORC1 is activated by Akt, we evaluated whether Akt overexpression reversed the negative effect of silmitasertib on mTORC1-dependent phosphorylation of S6K1. Overexpression of full-length wild-type Akt (Akt-WT) did not reverse the decrease in mTORC1 activity after inhibition of CK2 (Fig. 4c). CK2 is also described to phosphorylate Akt at Ser-129, which is important for the Wnt/ $\beta$-catenin pathway in HEK-293 cells $^{36,37}$. We analyzed whether overexpression of a mutant Akt unable to be phosphorylated by CK2 (AktS129A) had an effect on mTORC1 activity. The inability of being phosphorylated by CK2 slightly precluded AktS129A of activating mTORC1 (Fig. 4d and Supp Fig. 3A), which probably affected its capability to increase viability (Fig. 4e), however, it did not affect the augmented migration of Akt-S129A-expressing cells as compared to Akt-WT cells (Fig. 4f). This suggests that Ser-129 is somehow important for promoting viability of DLD-1 cells by probably promoting a full activation of mTORC1, which is evidenced for a slight decrease of pT389-S6K1. However, this residue would be expendable for the PI3K/ Akt-promoted migration.

Nutrients and growth factors, as well as altered PI3K/ Akt signaling, have been shown to promote subcellular 


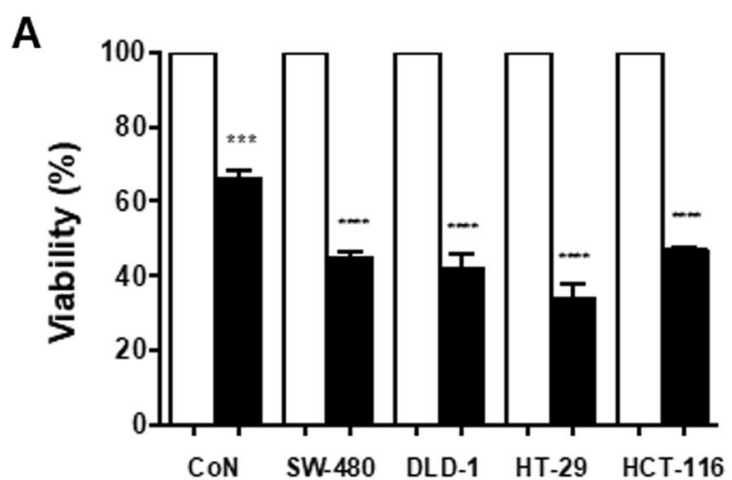

B
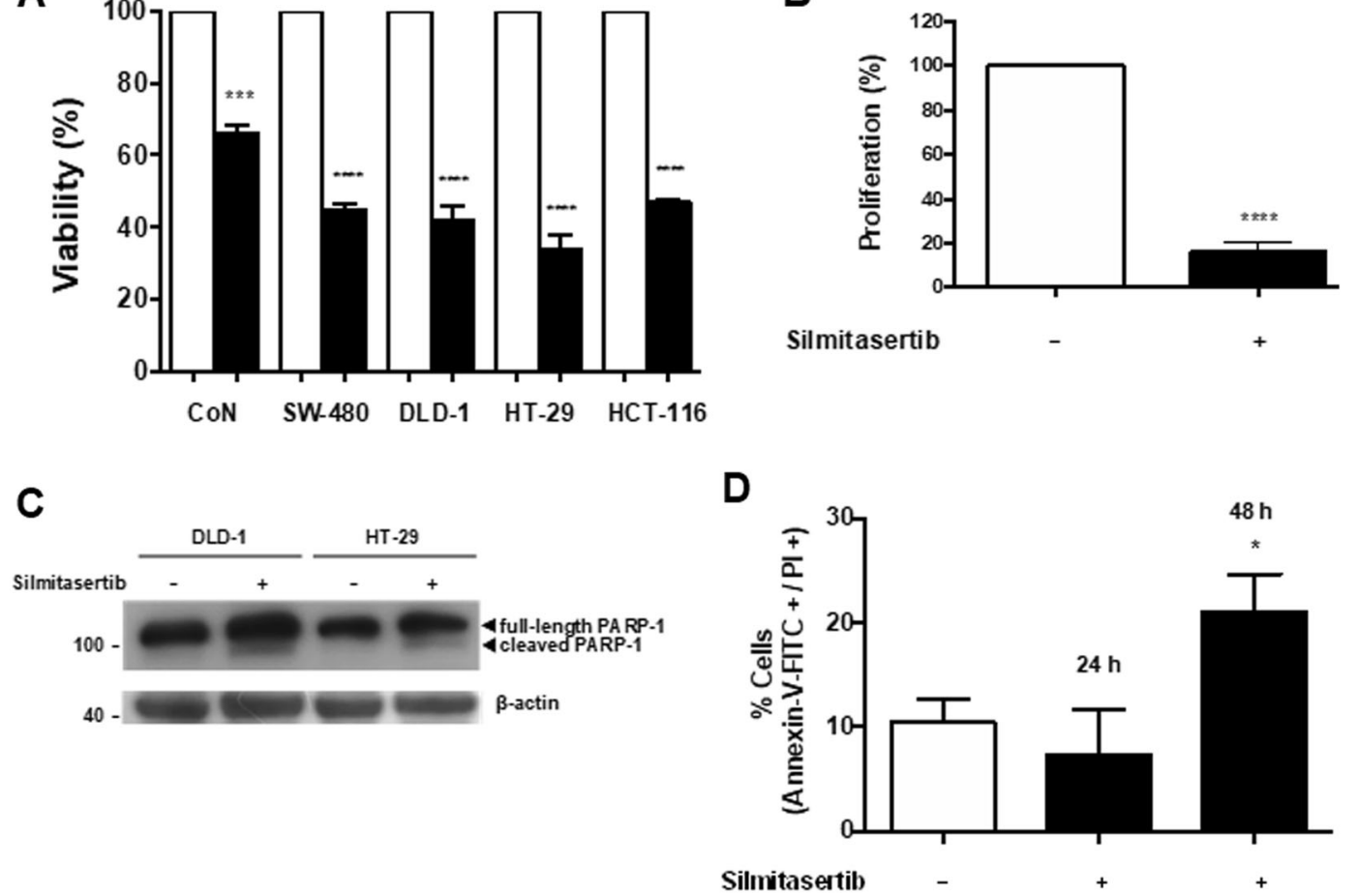

Fig. 2 Silmitasertib decreases viability and proliferation of CRC cells. a Cell viability was determined by MTS assay in normal (CoN) and CRC cells (SW-480, DLD-1, HT-29, and HCT-116) after incubation with either vehicle (white bars) or $25 \mu \mathrm{M}$ silmitasertib (black bars) for $48 \mathrm{~h}$. $\mathbf{b}$ DLD-1 cells were treated as in $\mathbf{a}$ and proliferation was determined by CFSE staining and cytometry. c Protein levels of caspase-cleaved PARP-1 were detected by western blot in DLD-1 and HT-29 cells treated as in a. d DLD-1 cells treated with $25 \mu \mathrm{M}$ silmitasertib for 24 and $48 \mathrm{~h}$ were analyzed through double positive annexin-V-FITC/PI staining by cytometry. Data represents the average \pm SEM $(n=3)$; ${ }^{*} p<0.05$

localization of mTORC1 to the outer layer of lysosomal membranes, where it is activated by Rheb ${ }^{6,8,9}$. We evaluated whether silmitasertib affected the localization of mTORC1 at lysosomes of DLD-1 cells. As early as $6 \mathrm{~h}$, untreated cells showed perinuclear localization of lysosomes with the expected colocalization of mTORC1, while in treated cells lysosomes were more disperse and apparently smaller, and their colocalization with mTORC1 was reduced (Supp Fig. 3B), suggesting that CK2 inhibition hindered localization of mTORC1 to lysosomes and probably its activation by Rheb. MTORC1 is also known to be a key regulator of autophagy ${ }^{38}$. In fact, mTORC1 inhibition has been linked to augmented autophagy in different cancer cell lines ${ }^{8,19,39}$. Lysosomal Rheb-activated mTORC1 inhibits ULK-1 by phosphorylation at Ser757, which is key for early autophagy induction $^{13,14}$. Thus, augmented pSer757-ULK-1 levels are a good marker of autophagy blockage. Therefore, to assess whether CK2 inhibition could have an impact on the activation of ULK-1, cells were treated with silmitasertib for $6 \mathrm{~h}$, following analysis of mTORC1 and ULK-1 phosphorylation by western blot. As expected, silmitasertib led to decreased levels of mTORC1-dependent phosphorylation in both targets, pT389-S6K1 and
pS757-ULK-1 (phospho-inhibitory site), thus meaning that $\mathrm{mTORC} 1$ and ULK-1 were inhibited and more active (i.e., less-inhibited), respectively, as compared with untreated cells (Supp Fig. 3C). Altogether, these results indicate that CK2 inhibition may lead to inactivation of mTORC1 in CRC cells.

\section{Silmitasertib-dependent mTORC1 inhibition leads to formation of large vacuoles}

The onset of autophagy by ULK- 1 activation normally leads to phagophore formation, which then fuses with lysosomes to form an autophagolysosome ${ }^{13}$. Interestingly, massive large cytosolic vacuoles formed only in CRC cell lines treated for $6 \mathrm{~h}$ with silmitasertib and not in normal CoN cells (Fig. 5a and Supp Fig. 4A). In DLD-1 cells, a progressive dose-dependent increase of small $(<3 \mu \mathrm{m})$ vacuoles was observed with silmitasertib up to $12 \mathrm{~h}$. At higher times, the number of vacuoles decreased concomitant with the increase in silmitasertib concentration while displaying an increase in their size, suggesting a fusion of small vacuoles to form bigger structures of almost 5-6 $\mu \mathrm{m}$. At both higher silmitasertib concentrations and treatment times, a cellular collapse was evidenced by large birefringent structures, indicative of 
massive cell death (Fig. 5b-d). In addition, viability decreased in a time-dependent manner, showing

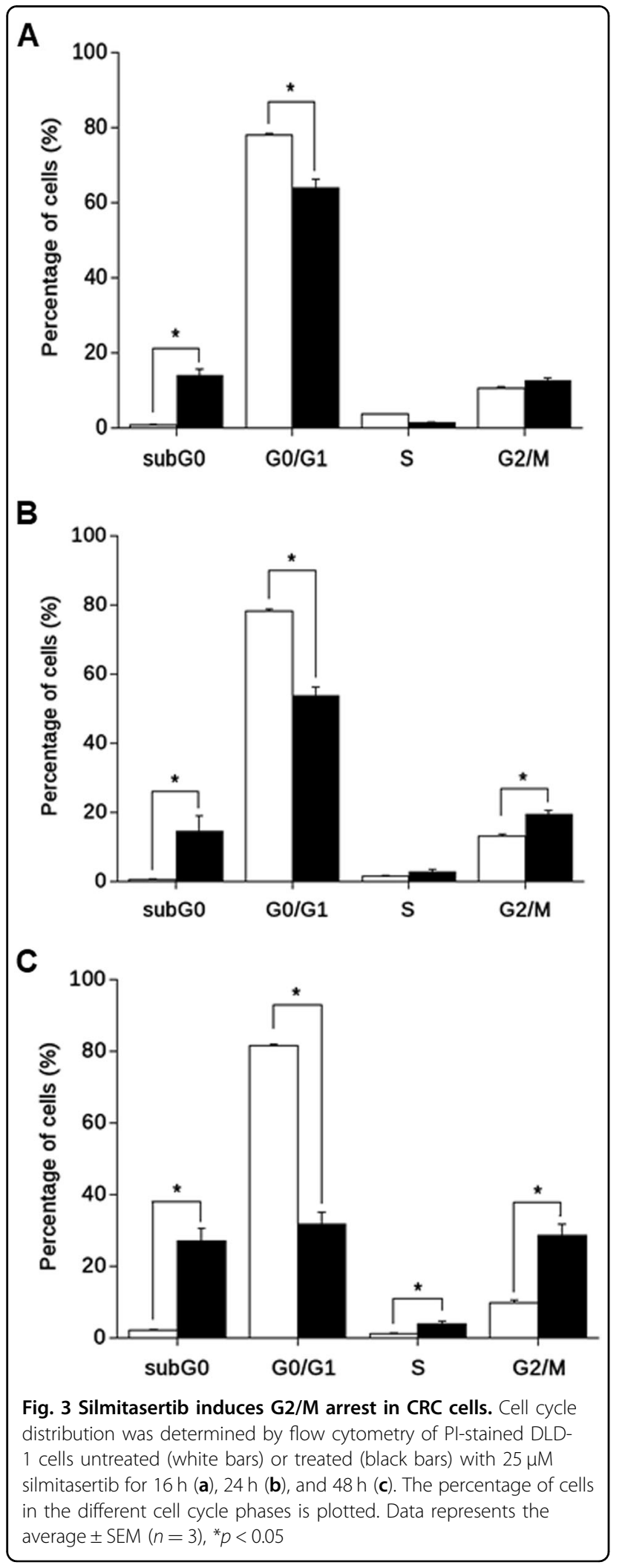

significance after $12 \mathrm{~h}$ (Supp Fig. 4B). Interestingly, the number of small $(<2 \mu \mathrm{m})$ vacuoles progressively increased up to $24 \mathrm{~h}$, while significantly dropping at $48 \mathrm{~h}$, which correlated with a significant increase of large $(>4.5 \mu \mathrm{m})$ vacuoles. To confirm that the effect of silmitasertib on vacuole formation is caused by specific inhibition of CK2, expression of CK2 $\alpha$ was silenced with the same siRNA as above (see Fig. 4b). Thus, CK2 silencing was sufficient to produce massive vacuole formation $(\approx 4 \mu \mathrm{m})$, which progressed both in number and size in time, reaching larger structures $(>5 \mu \mathrm{m})$ after $24 \mathrm{~h}$ of cell growth, resembling those triggered by silmitasertib at either $25 \mu \mathrm{M}$ for $48 \mathrm{~h}$ or $50 \mu \mathrm{M}$ for $24 \mathrm{~h}$ (Fig. 5e). As control, DLD-1 cells were treated with $100 \mathrm{ng} / \mathrm{ml}$ nocodazole for $24 \mathrm{~h}$, which was unable to preclude the silmitasertib-dependent massive vacuolization and thus discarding that this process is product of a G2/M arrest (Supp Fig. 4C).

To investigate whether those vacuoles correspond to autophagolysosomes, DLD-1 cells were loaded with lysotracker, which fluoresces in acidic pH like in lysosomes. As observed in Fig. 6a, vacuoles formed by treatment with silmitasertib were marked with lysotracker, indicating an acidic inner environment and which, due to their large size, could correspond to autophagolysosomes. During autophagy, ULK-1 phosphorylates and activates a class III PI3K, promoting the docking of the ubiquitin-like factor LC3-II to autophagolysosome membranes, which binds to its receptor p62 and together are ultimately degraded at the expanding autophagolysosome ${ }^{13}$. Thus, to assess whether silmitasertib-dependent ULK-1 activation and vacuolization are related to induction of autophagy, GFP-LC3 protein was expressed in DLD-1 cells and its subcellular distribution was evaluated after treatment with silmitasertib up to $12 \mathrm{~h}$. Unexpectedly, untreated control cells showed GFP-tagged structures suggesting docking of LC3 at autophagolysosomes, which diminished after treatment for $6 \mathrm{~h}$ and almost completely after $12 \mathrm{~h}$. Importantly, large vacuoles already present at $6 \mathrm{~h}$ did not recruit GFP-LC3 to their membranes at $12 \mathrm{~h}$ (Fig. $6 \mathrm{~b}$ and Supp Fig. 5A). Indeed, similar results were obtained in HeLa cervical cancer cells, where large vacuoles formed under treatment with silmitasertib, which were also GFPLC3-negative (Supp Fig. 5B). Additionally, decreased LC3-II and p62 levels were observed in DLD-1 cells treated with silmitasertib and bafilomycin-A1, an inhibitor of autophagic flow, for a period of up to $18 \mathrm{~h}$ (Fig. 6c, d). Importantly, this phenomenon was not related to de novo expression of representative autophagy genes, such as Beclin-1 and LC3 (Supp Fig. 5C) and vacuole formation was not blocked by inhibition of autophagy with 3methyladenine (Supp Fig. 5D), inhibition of apoptosis with Z-VAD-FMK (Supp Fig. 5E), or inhibition of necroptosis with Nec1s (Supp Fig. 5F). Thus, our results suggest that silmitasertib-dependent mTORC1 inhibition, 

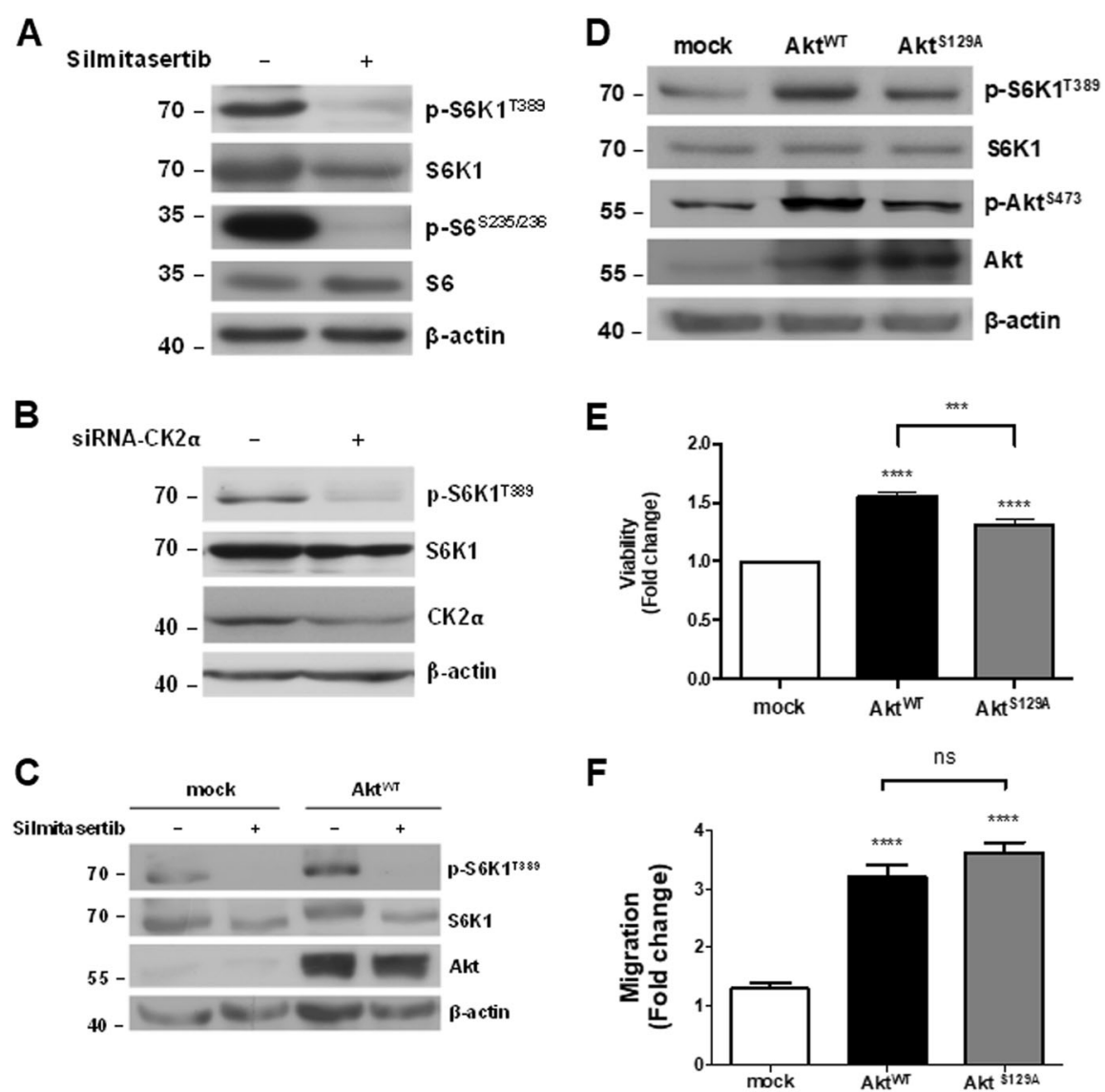

Fig. 4 Decreased mTORC1 activity in CRC cells treated with silmitasertib. a DLD-1 cells were incubated with $25 \mu \mathrm{M}$ silmitasertib for $16 \mathrm{~h}$. The levels of the indicated proteins were analyzed by western blot. b Cells were transfected with $100 \mathrm{nM}$ of a specific siRNA for CK2a. The indicated proteins were analyzed by western blot. c Cells were transfected with the plasmid pCMV6-myr-AKT-1-CA-HA and then treated with $25 \mu \mathrm{M}$ silmitasertib for $16 \mathrm{~h}$. Protein levels were analyzed by western blot. $\mathbf{d}$ Levels of indicated proteins in cells transfected with the plasmids pCMV6-myrAKT-1-CA-HA or pCMV6-myr-AKT-1-S129A-HA were analyzed by western blot. e Cell viability determined by MTS assay of cells transfected as in d. $\mathbf{f}$ Cell migration determined by transwell assay of cancer cells transfected as in $\mathbf{d}$. Data represents the average \pm SEM $(n=3) ;{ }^{* *} p<0.001 ;{ }^{* * * *} p<$ 0.0001; ns: not significant

despite leading to the formation of acidic large LC3negative vacuoles, they do not derive from induction of either autophagy, apoptosis or necroptosis.

\section{Vacuoles derive from a massive silmitasertib-dependent macropinocytosis}

Since the large acidic vacuoles induced by silmitasertib do not have LC3 on their membranes, they could correspond to an endosomal compartment distinct from autophagolysosomes. Interestingly, we observed that silmitasertib-dependent vacuolization is precluded by bafilomycin-A1 treatment (Supp Fig. 5A), which has been shown to impair the macropinocytosis pathway elsewhere $^{40}$. Moreover, the late endosomal marker Rab7 has been reported as an indicator of macropinosomes ${ }^{41,42}$. Indeed, early large vacuoles formed after treatment with silmitasertib were significantly Rab7 positive at $6 \mathrm{~h}$ (Fig. 7a). Similarly, another late endosomal marker,
LAMP1, was also located at the membrane of such vacuoles after treatment with silmitasertib (Supp Fig. 6). Therefore, silmitasertib-promoted vacuoles in CRC cells correspond to an endolysosomal compartment similar to macropinosomes.

Because macropinocytosis is an endocytic pathway that mediates non-selective uptake of solutes, we used dextran to confirm the endocytic nature of the silmitasertibderived vacuoles. An internalization assay was performed using $10 \mathrm{kDa}$ FITC-conjugated dextran. Cells were treated with silmitasertib during $6 \mathrm{~h}$ and then incubated with dextran for $1 \mathrm{~h}$. Consistently, control cells showed a constitutive level of endocytosis, however, the green fluorescence was concentrated at the large vacuoles observed following treatment with silmitasertib (Fig. 7b). Taken together, these results strongly suggest that inhibition of CK2 with silmitasertib leads to massive macropinocytosis in CRC cells. 


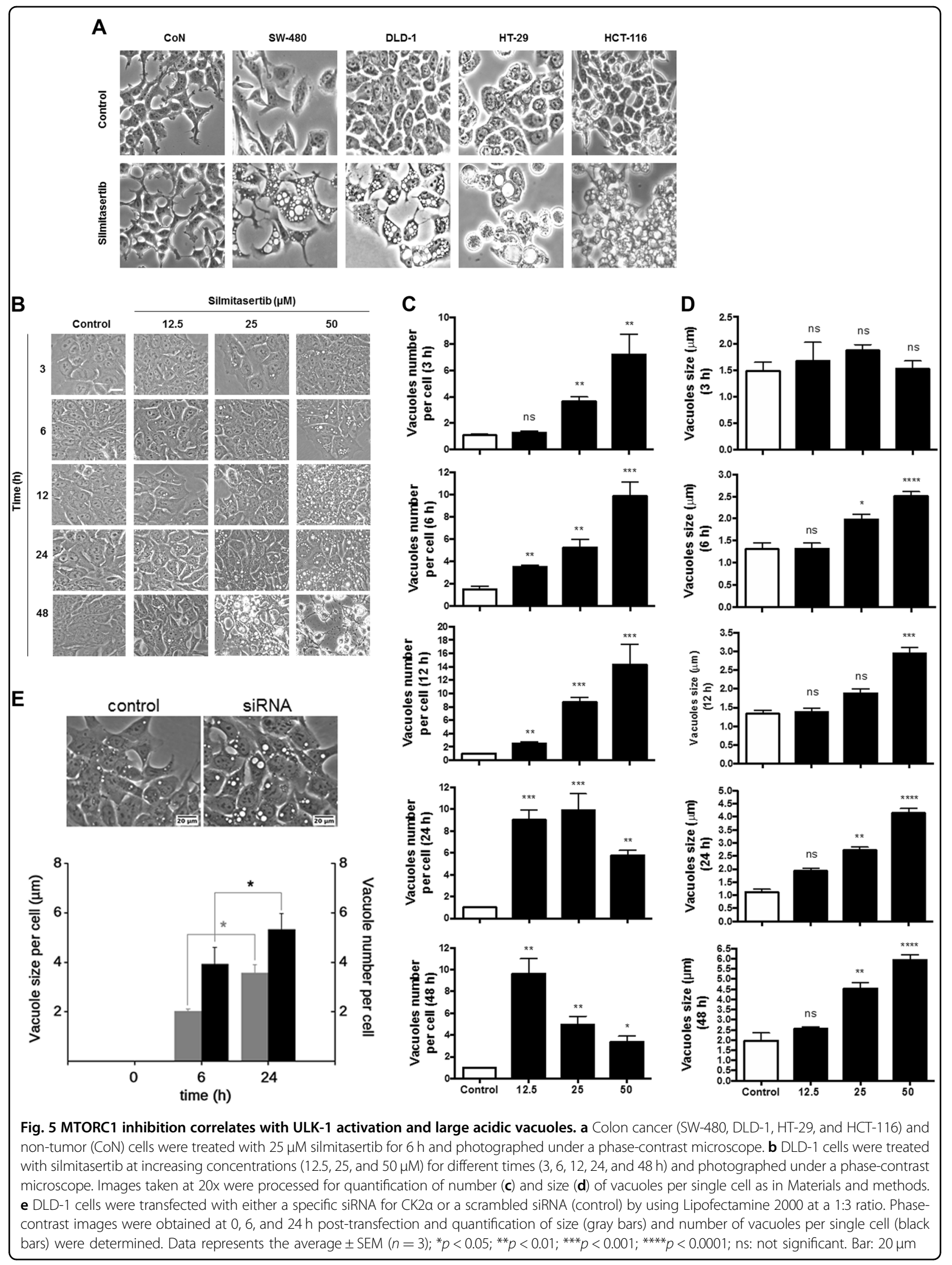




\section{Discussion}

Protein kinase CK2 is elevated in different cancers including CRC and its expression and activity are strongly linked to hyperproliferation and resistance to apoptosis, suggesting an important role in tumorigenesis ${ }^{21}$. In fact, CK2 has been proposed as a putative prognostic marker in cancer $^{43-45}$. Consistently, an idea has emerged that some tumor cells become "CK2-addicted" in order to create a permissive microenvironment for tumor progression and to eventually favor an aggressive phenotype ${ }^{23}$. Thus, inhibition of CK2 should be extremely catastrophic in those cancer cells. Therefore, CK2 has evolved as an attractive therapeutic target in cancer, with two pharmacological inhibitors extensively used in in vitro and ex vivo experiments, TBB (4,5,6,7-tetrabromobenzotriazole) and silmitasertib (formerly CX-4945), the latter having been tested in clinical trials. Both are CK2 competitive inhibitors for the ATP binding site, normally used at the micromolar scale by us and other groups ${ }^{46,47}$. However, silmitasertib had a more potent effect than TBB in decreasing viability of CRC cells, since a four-fold lower concentration of the former was used in this work to achieve the same effect (data not shown). Indeed, DLD-1 cells showed significant decrease in in vitro tumorigenesis after 21 days, accounting for an extremely deleterious effect of silmitasertib on cell viability. This correlated with early cell cycle arrest at G2/M, previously seen in breast, lung and glioblastoma cancer cells ${ }^{27,48,49}$. Of note, the silmitasertib-dependent arrest in our DLD-1 cells was distinct to what we observed in HT-29 cells treated with TBB for $24 \mathrm{~h}^{35}$. Also relevant, the G2/M arrest was irrespective of activating mutations in the PIK3CA gene, a common trait found in CRC tumors ${ }^{2}$, since the same was observed in SW-480 cells, which are free of the genomic alteration. This suggests a specific and broad effect of silmitasertib on any CRC cell, endorsing its use in CRC patient therapy in the future.

Our data show that CK2 may activate the PI3K/Akt/ mTORC1 signaling pathway in CRC. It has been described that silmitasertib inhibits this pathway and hence decreases viability of lung cancer cells ${ }^{27}$. Additionally, silmitasertib in combination with erlotinib (an EGFR inhibitor) produces a complete inhibition of this pathway, inducing apoptosis in squamous lung cancer cells ${ }^{28}$. Moreover, two pharmacological inhibitors of mTORC1, everolimus and temsirolimus, exemplify the therapeutic importance of the inhibition of this pathway in patients with advanced renal carcinoma $^{50}$. Thus, taking into consideration our published findings on the functional relationship between CK2 and $\mathrm{Akt}^{36,37}$, an attractive possibility emerged where CK2 may eventually upregulate mTORC1 via phosphorylation/ activation of Akt in CRC cells. However, overexpression of the CK2-phosphorylation resistant mutant, Akt-S129A, behaved similarly to wild-type Akt on mTORC1 activity.
This could be a consequence of an mTORC1-independent effect since activation of mTORC1 by Akt involves different proteins. For example, CK2 may alter the activity of the Rags proteins, which are responsible for mTORC1 anchorage at the lysosome membrane ${ }^{51}$.

Silmitasertib-dependent inhibition of mTORC1 has been seen to promote autophagy in lung, pancreas and glioblastoma cancer cells, where inhibition of CK2 led to inhibition of mTORC1 and displayed features of autophagy $^{26,27,52}$. Interestingly, it has also been described that a decrease in the expression and activity of CK2 leads to the formation of large vacuoles in pancreatic cancer cells, related to decreased viability and autophagy induction ${ }^{26}$. Consistent with this, we observed the formation of early large acidic vacuoles in DLD-1 cells treated with silmitasertib. In fact, these vacuoles had been previously seen in CRC cells following incubation with other less-potent CK2 inhibitors, including apigenin and DRB, with no explanation about their origin (Tapia J.C., personal communication). These vacuoles lacked LC3 on their membranes and the LC3-II and p62 levels did not correlate with early autophagy induction ${ }^{53}$. Instead, silmitasertib inhibited accumulation of LC3-II and p62 in presence of bafilomycin-A1, indicative of autophagy inhibition. This result is contradictory, considering that inhibition of CK2 with silmitasertib-promoted ULK-1 activation associated to the inhibition of mTORC1. Although it is not possible to rule out that the large vacuoles induced by silmitasertib somehow mask an induction of autophagy, CK2 may have a regulatory effect (e.g., by phosphorylation) on some key autophagy regulators, leading finally to an attenuation of autophagy induction after mTORC1 activation. In fact, p62 is phosphorylated by CK2 at Ser-403, thereby favoring autophagic clearance of ubiquitinated targets ${ }^{54}$. Likewise, ULK-1 contains putative phosphorylation sites for CK2, one (Thr-109) located in its kinase domain, although if the site(s) are functionally relevant for autophagy is yet unknown.

Silmitasertib is a well-known inducer of cell death in different cancer cells, including CRC, although the underlying mechanism not only involves apoptosis but also other non-apoptotic mechanisms. The difficulty for precisely determine the death mechanism is probably due to the multiple targets that CK2 phosphorylates involved in several cancer-related processes ${ }^{23}$. Here, we determined that the viability of CRC cells treated with this inhibitor decreased $60-70 \%$, of which only a $20 \%$ correspond to apoptosis at $48 \mathrm{~h}$. However, autophagy and apoptosis are related cellular mechanisms. When autophagy is deficient or inhibited, apoptosis is induced, and conversely autophagy is induced upon deficient apoptosis ${ }^{15}$. This crossregulation responds to the interaction of two regulatory proteins, Beclin-1 (autophagy inducer) and Bcl-2 (antiapoptotic $)^{55}$, which leads to the inhibition of autophagy- 
A
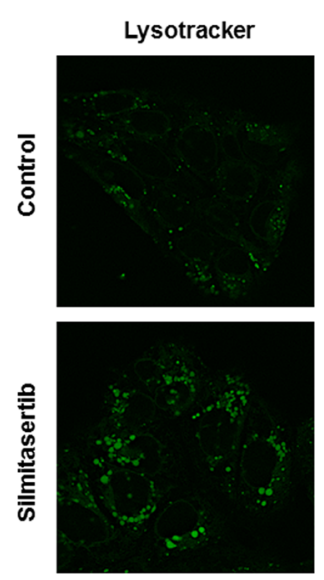

B
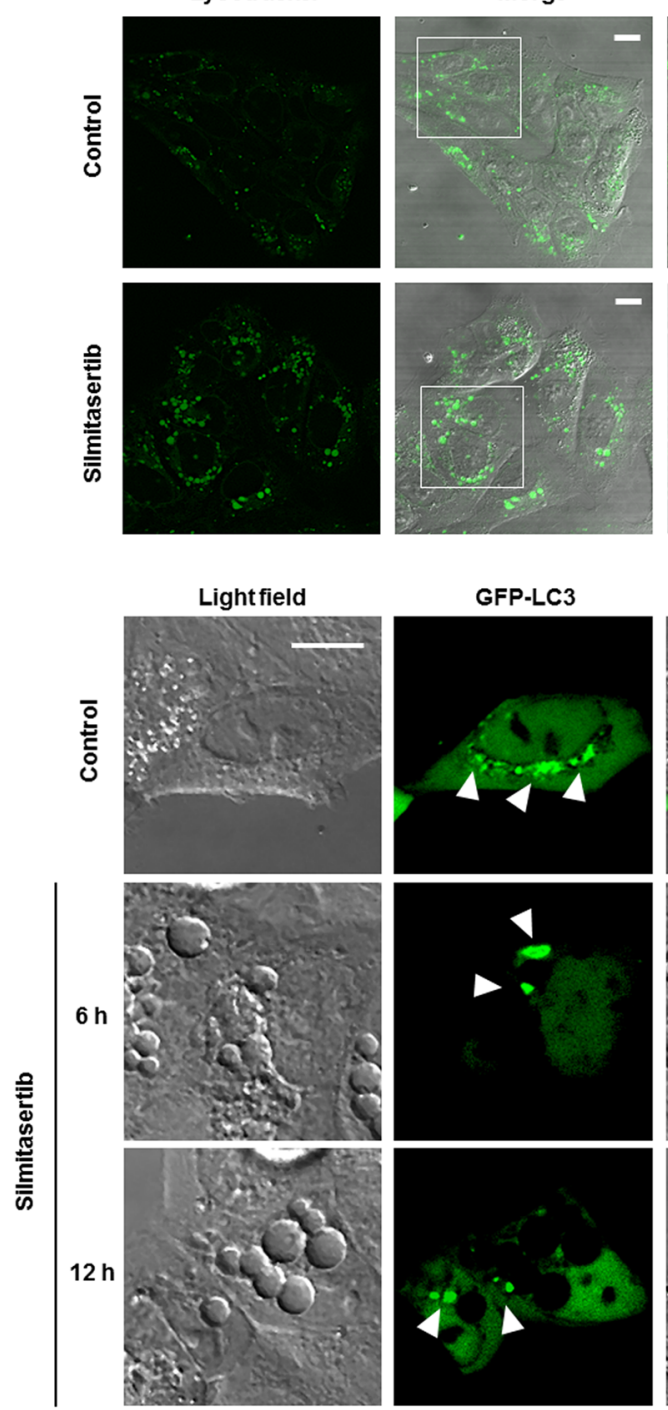
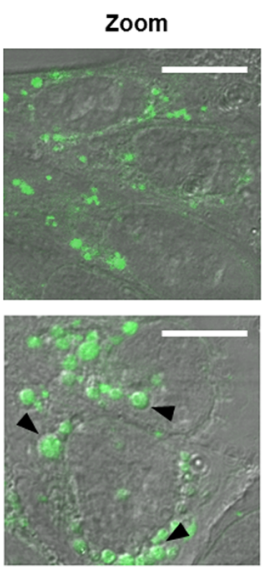

C

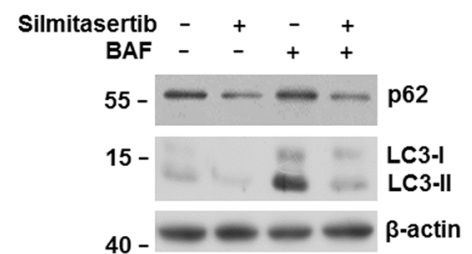

D

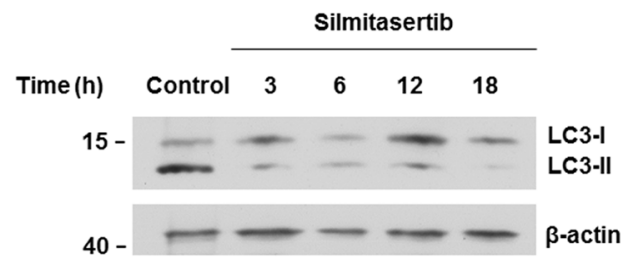

Fig. 6 Silmitasertib-derived vacuoles are acidic but negative for LC3-II and p62. a DLD-1 cells treated with $25 \mu \mathrm{M}$ silmitasertib for $6 \mathrm{~h}$ were then labeled with $1 \mu \mathrm{M}$ lysotracker (green) for $30 \mathrm{~min}$ and living cells were visualized by video-microscopy. Black arrowheads indicate the vacuoles highlighted in white squares. Representative images are shown $(n=3)$, scale bars: $10 \mu \mathrm{m}$. b DLD-1 cells were transfected with a plasmid encoding GFP-LC3. After a $24 \mathrm{~h}$ transfection, cells were treated with $25 \mu \mathrm{M}$ silmitasertib for 6 or $12 \mathrm{~h}$ and visualized by confocal microscopy. White arrowheads indicate LC3 puncta. c DLD-1 cells were treated during $6 \mathrm{~h}$ with $25 \mathrm{\mu M}$ silmitasertib and/or $100 \mathrm{nM}$ bafilomycin-A1 (BAF), followed by analysis of LC3$\mathrm{I} / \mathrm{II}$ and p62 levels by western blot. $\mathbf{d}$ DLD-1 cells were treated with $25 \mu \mathrm{M}$ silmitasertib and the last $3 \mathrm{~h}$ with $100 \mathrm{nM}$ BAF, then cultured for another 3 , 6,12 , and $18 \mathrm{~h}$, followed by analysis of LC3-I/II levels by western blot 


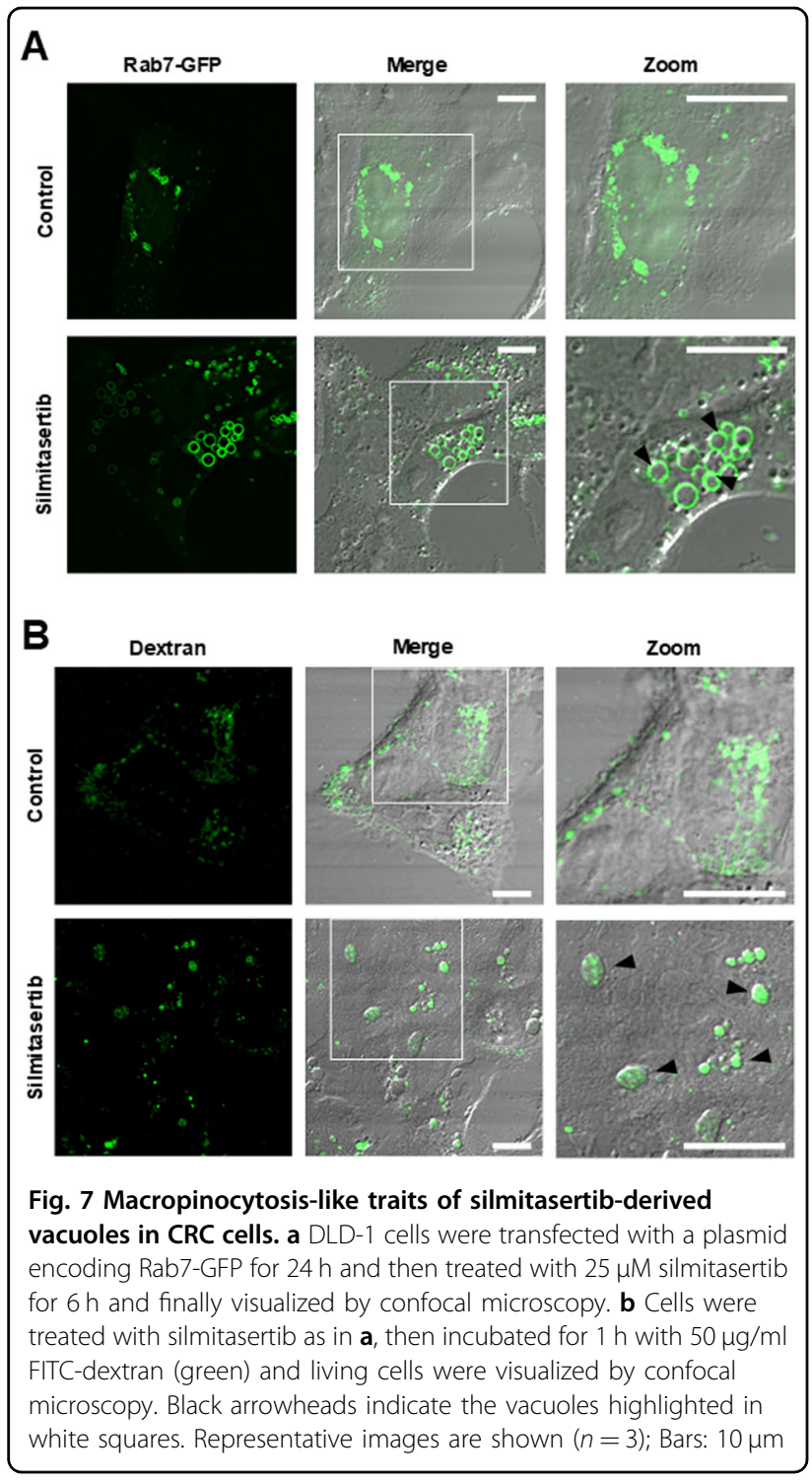

associated death. Although interesting, this mechanistic relationship was not addressed in this work and the observed cell death remains of unknown origin.

Our findings indicate that silmitasertib induces a deep change in the endocytic pathway of CRC cells, where large acidic $\mathrm{LC}^{-}-\mathrm{II}^{-} / \mathrm{LAMP}^{+} / \mathrm{Rab}^{+}$macropinosomes are massively formed (see Fig. 8). Indeed, recent data in literature support this mechanism, since some antineoplastic drugs promote massive vacuolization that eventually leads to necrotic-like cell death, often named methuosis $^{42,56-58}$. Interestingly, the mitogen-activated protein kinase kinase 4 (MKK4) has been indicated as a key factor in macropinocytosis activation ${ }^{58}$ and its downregulation is associated with liver metastasis in $\mathrm{CRC}^{59}$. Moreover, silencing of the CK2 $\alpha$ subunit leads to MKK4 activation and, consequently, significant death of PANC-1 human pancreatic cancer cells treated with gemcitabine $^{60}$, which displays methuosis-like traits. Nevertheless, whether the putative CK2-dependent regulation of MKK4 is involved in CRC cell death as well as decreased in vitro tumorigenesis after 21 days, is an issue that warrants further research. Altogether, our findings point to this selective inhibitor of CK2 as a promissory therapeutic drug for CRC patients and probably other CK2-addicted cancers.

\section{Materials and methods \\ Cell culture}

Human normal colon cells CCD $841(\mathrm{CoN})$ and colon cancer cell lines (SW-480, DLD-1, HT-29 and HCT-116) were purchased from ATCC. Cells were maintained at 37 ${ }^{\circ} \mathrm{C}$ and $5 \% \mathrm{CO}_{2}$ in RPMI-1640 medium (Invitrogen, Paisley, UK) supplemented with 10\% Fetal Bovine Serum (HyClone, Logan, UT), antibiotics $(10000 \mathrm{U} / \mathrm{ml}$ penicillin and $10 \mu \mathrm{g} / \mathrm{ml}$ streptomycin) and eventually treated with silmitasertib (ApexBio Technology LLC, Houston, TX).

\section{Expression vectors and transfection}

The pCMV6-myr-AKT-1-CA-HA plasmid ${ }^{61}$ was purchased from Addgene (Cambridge, USA) and pCMV6myr-AKT-1-S129A-HA was a kind gift from Dr. Maria Ruzzene $^{25}$. Expression vectors for Rab7-GFP, LAMP1RFP, GFP-LC3, HA-CK $2 \alpha$ and GFP-CK $2 \alpha$ have been described elsewhere ${ }^{17,62}$. Small interfering RNAs (siRNA) targeted to CK2 $\alpha$ and control siRNA were purchased from Santa Cruz Biotechnology (Santa Cruz, CA). Transfections were carried out with $2 \mu \mathrm{g}$ each DNA by using Lipofectamine 2000 as reagent, according to the manufacturer's instructions (Life Technologies, Rockford, IL).

\section{Western blot}

Cells were lysed in RIPA buffer (Thermo Scientific, Rockford, IL) with the cocktail of protease and phosphatase inhibitors PhoSTOP (Roche, Mannheim, Germany). Proteins were separated by sodium dodecyl sulfate polyacrylamide gel electrophoresis, transferred to NitroPure membranes (Macherey-Nagel, Düren, Germany) and blocked with 5\% BSA (Thermo Scientific, Rockford, IL) in PBS/0.1\% Tween. Blots were incubated with primary antibodies (Cell Signaling Technology, Beverly, MA): rabbit polyclonal anti-PARP (1:1000), rabbit polyclonal anti-CK2 $\alpha$ (1:1000), rabbit polyclonal anti-LC3 (1:1000), rabbit monoclonal anti-pSer757-ULK-1 (1:1000), rabbit monoclonal anti-ULK-1 (1:1000), rabbit monoclonal antiAkt (1:1000), rabbit monoclonal anti-pThr389-S6K1 (1:1000), rabbit polyclonal anti-S6K1 (1:1000), rabbit polyclonal anti-pSer235/Ser236-S6 (1:1000) and mouse monoclonal anti-S6 (1:1000). Mouse monoclonal anti-p62 (1:1000) was obtained from BD Biosciences and anti-actin (1:2000) from Santa Cruz Biotechnology (Santa Cruz, 


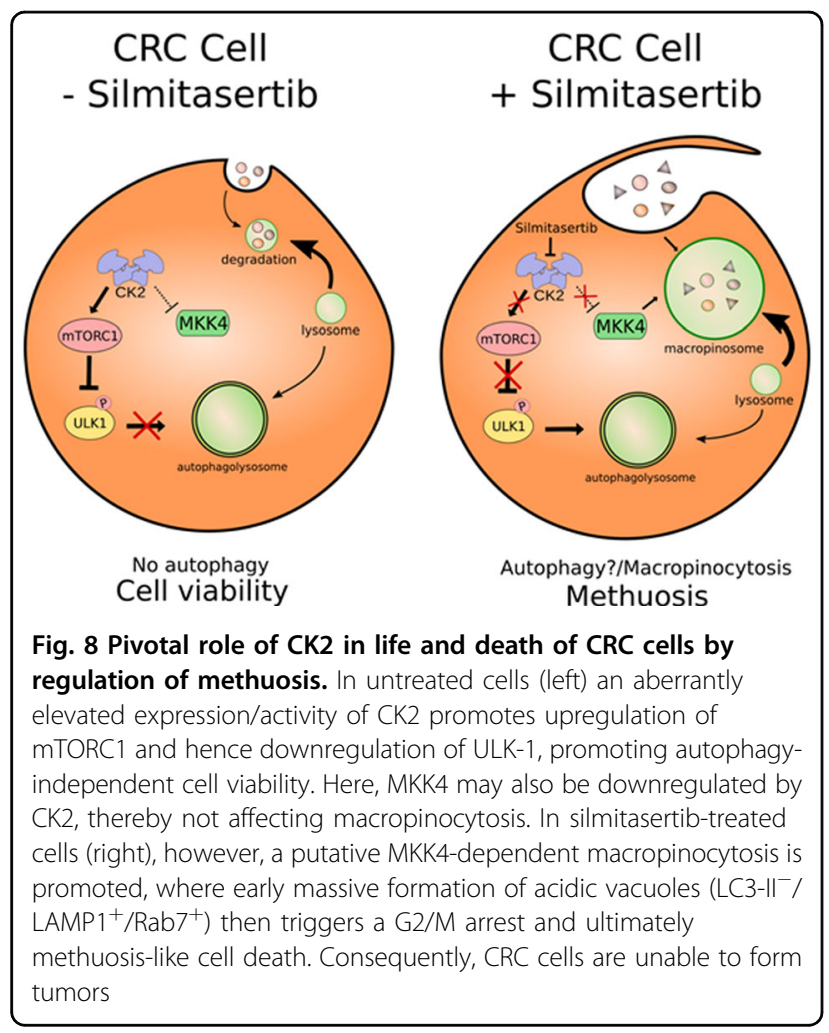

CA). The secondary antibodies used were: anti-goat IgGHRP (1:2000), anti-rabbit IgG-HRP (1:2000) and antimouse IgG-HRP (1:2000) from Santa Cruz Biotechnology. Blots were revealed with the EZ-ECL chemiluminescent kit (Biological Industries, Haemek, Israel).

\section{Immunofluorescence and confocal microscopy}

Cells were grown on glass coverslips for the indicated times, washed with PBS, fixed in $3.7 \%$ p-formaldehyde for $15 \mathrm{~min}$, permeabilized for $10 \mathrm{~min}$ at room temperature (RT) in $0.2 \%$ Triton X-100/PBS (i.e., PBST), blocked with $3 \% \mathrm{BSA} / \mathrm{PBST}$ for $30 \mathrm{~min}$ at RT and incubated with primary antibody in 1\% BSA/PBST (1:200 for anti-mTOR and 1:200 for anti-HMGB1) for $1 \mathrm{~h}$ at RT. Incubation with secondary antibody (1:500) was performed in 1\% BSA/ PBST for $45 \mathrm{~min}$ at RT in the dark. Lysosomes were stained with $1 \mu \mathrm{M}$ lysotracker for $30 \mathrm{~min}$ and nuclei with DAPI for $10 \mathrm{~min}$ (Thermo-Fisher Scientific). For dextran uptake, cells were incubated for $1 \mathrm{~h}$ with $50 \mu \mathrm{g} / \mathrm{ml}$ FITCconjugated dextran (Thermo-Fisher Scientific). For some experiments, slides were mounted in ProLong ${ }^{\circledR}$ (Life Technologies) and images obtained with an OlympusIX81 DSU Spinning Disk confocal microscope. For videomicroscopy experiments, cells seeded on glass coverslips were mounted on a microscope chamber equipped with a heating unit $\left(37^{\circ} \mathrm{C}\right)$ and $5 \% \mathrm{CO}_{2}$, and then analyzed by fluorescence microscopy using an Olympus Confocal FV1000. The program FV10-ASW 3.0 was used for all acquisitions and settings. For determination of number and size of vacuoles, images were taken at 20x magnification, segmented and binarized on the ImageJ software by homemade commands, and put all together in a macro file. By using the /Window sincronization function on ImageJ, circular regions corresponding to vacuoles were tracked on binary images by contrasting them with the original ones. Size (diameter) and number of vacuoles identified were automatically measured by the /Multi measure function on ImageJ.

\section{Viability}

Cells growing at $50-60 \%$ confluency on six-well plates were transfected and/or treated with inhibitor as indicated in the figure, left in culture during $24 \mathrm{~h}$ and subsequently re-plated in 96-well plates at a density of $1 \times 10^{4}$ cells per well. Viability was measured using the MTS $^{\oplus}$ assay according to the manufacturer's instructions (Promega).

\section{Flow cytometry}

For proliferation assay, cells were detached by trypsinization, washed and left in PBS to a density of $10^{6}$ cells/ $\mathrm{ml}$. CFSE (eBioscience) was added to a final concentration of $5 \mu \mathrm{M}$, mixed for $10 \mathrm{~s}$ and incubated for $10 \mathrm{~min}$. One volume of FBS was added, followed by complete medium and cells were then pelleted, washed, seeded into p60 petri dishes, and incubated with DMSO (dimethyl sulfoxide) or silmitasertib for $48 \mathrm{~h}$. For apoptosis assay, cells $\left(2 \times 10^{5}\right)$ were washed with PBS and left in a total volume of $100 \mu \mathrm{l}$ binding buffer $\left(2.5 \mathrm{mM} \mathrm{CaCl}_{2}, 0.01 \mathrm{M}\right.$ Hepes, $0.14 \mathrm{M}$ $\mathrm{NaCl}$ ). One microliter annexin-V-FITC (Biolegend) and $2 \mu \mathrm{l} 50 \mu \mathrm{g} / \mathrm{ml}$ propidium iodide (PI) were added and cells incubated for $15 \mathrm{~min}$ at RT. Finally, $300 \mu \mathrm{l}$ binding buffer was added to stop the reaction. For cell cycle assay, cells were incubated with $25 \mu \mathrm{M}$ silmitasertib for the indicated times and then centrifuged at $1000 \times \mathrm{g}$ for $5 \mathrm{~min}$ at RT, washed in $1 \mathrm{ml}$ cold PBS, suspended in $1 \mathrm{ml}$ staining solution containing $0.1 \%$ Triton X-100, $50 \mu \mathrm{g} / \mathrm{ml}$ PI and $200 \mu \mathrm{g} / \mathrm{ml}$ RNAse, and incubated for $30 \mathrm{~min}$ at $37^{\circ} \mathrm{C}$ in the dark. All assays were analyzed on a Becton-Dickinson LSR Fortessa X-20 flow cytometer and the FACSDiva 8.02 software (San Jose, CA) at the MED.UCHILE-FACS Facility, Facultad de Medicina, Universidad de Chile.

\section{Anchorage-independent growth}

Cells $\left(2.5 \times 10^{3}\right)$ were suspended in $0.33 \%$ Bacto-agar (BD Biosciences) in a media containing $12.5 \%$ FBS in RPMI-1640. The cell suspension was then poured into 6well plates containing a layer of $2 \mathrm{ml} 0.5 \%$ agar in the same media. Plates were fed twice a week with $0.5 \mathrm{ml}$ RPMI-1640 supplemented with 10\% FBS. After 21 days, colonies were photographed under a Nikon Eclipse TS100 inverted microscope. Finally, cells were stained using $0.005 \%$ crystal violet dissolved in $20 \%$ methanol for $1 \mathrm{~h}$ at 
RT and colonies visible to the naked eye were also photographed and documented with a Nikon D5100 camera.

\section{Migration}

Migration assay was performed in Boyden Chambers of $6.5 \mathrm{~mm}$ diameter and $8 \mu \mathrm{m}$ pore, according to manufacturer's instructions (Transwell Costar). Briefly, the bottom of each insert was coated with $0.5 \mathrm{ml} 2 \mu \mathrm{g} / \mathrm{ml}$ fibronectin. Cells $\left(3.5 \times 10^{4}\right)$ were suspended in serumfree medium and seeded onto the top of each chamber insert. Medium supplemented with 10\% FBS was added to the bottom chamber. After $8 \mathrm{~h}$, inserts were removed, washed, and cells adhered to the bottom side of the inserts were stained with $0.1 \%$ crystal violet in $20 \%$ methanol. The number of migrated cells was determined under a Nikon Eclipse TS100 inverted microscope.

\section{Reverse transcription quantitative PCR (RT-qPCR)}

Total RNA from cells was extracted by using the EZNA Total RNA Kit I (Omega bio-tek, Giorgia USA) and treated with DNase (DNA-free Kit Ambion, Life Technologies). RNA concentration was measured with NanoQuant Infinite M200 pro spectrophotometer (Tecan). Reverse transcription was performed by using the AffinityScript QPCR cDNA Synthesis Kit (Agilent Technologies, TX). Quantitative real-time PCR was performed in a StepOne real-time PCR system (Applied Biosystems) with SYBR Green PCR master mix (Thermo-Fisher Scientific, Vilnius, Lithuania). Determinations were performed in triplicate ( 40 cycles) and the relative abundance of each mRNA was determined by using the $2^{-\Delta \Delta c t}$ method and normalized with GAPDH. The limits of a 95\% confidence interval were determined to indicate variability of the mean ratios for each experiment. Primers used were: Beclin-1 Fw 5'-ACCGTGTCACCATCCAGG AA-3' and $\mathrm{Rv}$ 5'-GAAGCTGTTGGCACTTTCTGT-3'. LC3 Fw 5'-CCGTCGGAGAAGACCTTCAA-3' and Rv 5'-GCATAGACCATGTACAGGAA-3'; GAPDH Fw 5'GAGTCAACGGATTTGGTCGT-3' and Rv 5'-GACAA GCTTCCCGTTCTCAG-3'.

\section{Statistics}

All values were expressed as mean \pm SEM of three independent experiments. Data was analyzed using twotailed unpaired Student's $t$-test and ANOVA. $p$-value was set at a nominal level of 0.05 or less.

\section{Acknowledgements}

We thank Félix Urra, Hernán Huerta, and Bárbara Pesce (Universidad de Chile) Diego A. Rodriguez (St. Jude Children's Research Hospital), as well as Milagros López de Armentia (Universidad Nacional de Cuyo) for their valuable technical support in some cytometry, soft-agar, necroptosis and microscopy analysis. This work was supported by CONICYT-FONDECYT-Chile grants \#1140345 (to V. A.B.), \#1161219 (to F.A.), \#11150624 (to M.V.G.), \#1160731 (to A.F.C.) and \#1160889 (to J.C.T.); CONICYT-FONDAP-Chile grant \#15130011 (to F.A.); SeCTyP 06/J432 grant from Universidad Nacional de Cuyo and PICT2013-0305 grant from Agencia Nacional de Investigación Científica y Tecnológica, Argentina (to M.I.C.)

\section{Author details}

${ }^{1}$ Departamento de Oncología Básico Clínica, Facultad de Medicina, Universidad de Chile, Santiago, Chile. ${ }^{2}$ Fundación Ciencia \& Vida, Andes Biotechnologies SpA, Facultad de Ciencias de la Vida, Universidad Andrés Bello, Santiago, Chile. ${ }^{3}$ Centro de Investigación Biomédica, Facultad de Medicina, Universidad de Los Andes, Santiago, Chile. ${ }^{4}$ Departamento de Bioquímica y Biología Molecular, Facultad de Ciencias Biológicas, Laboratorio de Transducción de Señales y Cáncer, Universidad de Concepción, Concepción, Chile. ${ }^{5}$ Laboratorio de Biología Celular y Molecular, Instituto de Histología y Embriología, Facultad de Ciencias Médicas, CONICET, Universidad Nacional de Cuyo, Mendoza, Argentina. ${ }^{6}$ Present address: Laboratorio de Patología Molecular, Instituto de Bioquímica y Microbiología, Facultad de Ciencias, Universidad Austral de Chile, Valdivia, Chile

\section{Conflict of interest}

The authors declare that they have no conflict of interest.

\section{Publisher's note}

Springer Nature remains neutral with regard to jurisdictional claims in published maps and institutional affiliations.

Supplementary Information accompanies this paper at (https://doi.org/ 10.1038/s41419-019-1306-x).

Received: 8 June 2018 Revised: 20 November 2018 Accepted: 17 December 2018

Published online: 25 January 2019

\section{References}

1. Johnson, S. M. et al. Novel expression patterns of PI3K/Akt/mTOR signaling pathway components in colorectal cancer. J. Am. Coll. Surg. 210, 767-776 (2010). 776-768

2. Ligresti, G. et al. PIK3CA mutations in human solid tumors: role in sensitivity to various therapeutic approaches. Cell Cycle 8, 1352-1358 (2009).

3. Chowdhury, S. et al. Restoration of PTEN activity decreases metastases in an orthotopic model of colon cancer. J. Surg. Res. 184, 755-760 (2013).

4. Pópulo, H. et al. The mTOR signalling pathway in human cancer. Int. J. Mol. Sci. 13, 1886-1918 (2012).

5. Moschetta, M. et al. Therapeutic targeting of the mTOR-signalling pathway in cancer: benefits and limitations. Br. J. Pharmacol. 171, 3801-3813 (2014).

6. Laplante, M. \& Sabatini, D. M. mTOR signaling in growth control and disease. Cell 149, 274-293 (2012).

7. Dibble, C. C. \& Cantley, L. C. Regulation of mTORC1 by PI3K signaling. Trends Cell Biol. 25, 545-555 (2015).

8. Saxton, R. A. \& Sabatini, D. M. mTOR Signaling in Growth, Metabolism, and Disease. Cell 168, 960-976 (2017).

9. Menon, S. et al. Spatial control of the TSC complex integrates insulin and nutrient regulation of mTORC1 at the lysosome. Cell 156, 771-785 (2014).

10. Fujishita, T. et al. Inhibition of the mTORC1 pathway suppresses intestinal polyp formation and reduces mortality in ApcDelta716 mice. Proc. Natl Acad. Sci. USA 105, 13544-13549 (2008).

11. Deming, D. A. et al. mTOR inhibition elicits a dramatic response in PI3Kdependent colon cancers. PLOS ONE 8, e60709 (2013).

12. Russell, R. C. et al. Autophagy regulation by nutrient signaling. Cell Res. 24, 42-57 (2014).

13. Dunlop, E. A. \& Tee, A. R. mTOR and autophagy: a dynamic relationship governed by nutrients and energy. Semin. Cell Dev. Biol. 36, 121-129 (2014).

14. Jung, C. H. et al. mTOR regulation of autophagy. FEBS Lett. 584, 1287-1295 (2010).

15. Mathew, R. et al. Role of autophagy in cancer. Nat. Rev. Cancer 7, 961-967 (2007).

16. Dobashi, Y. et al. Mammalian target of rapamycin: a central node of complex signaling cascades. Int. J. Clin. Exp. Pathol. 4, 476-495 (2011). 
17. Campos, T. et al. Rheb promotes cancer cell survival through p27Kip1dependent activation of autophagy. Mol. Carcinog. 55, 220-229 (2016).

18. Coker-Gurkan, A. et al. Inhibition of autophagy by 3-MA potentiates purvalanol-induced apoptosis in Bax deficient HCT 116 colon cancer cells. Exp. Cell Res. 328, 87-98 (2014).

19. Wang, L. et al. Autophagy inhibition sensitizes WYE-354-induced anti-colon cancer activity in vitro and in vivo. Tumour Biol. 37, 11743-11752 (2016).

20. Yang, Z. et al. High expression of Beclin-1 predicts favorable prognosis for patients with colorectal cancer. Clin. Res. Hepatol. Gastroenterol. 39, 98-106 (2015).

21. St-Denis, N. A. \& Litchfield, D. W. Protein kinase CK2 in health and disease: From birth to death: the role of protein kinase CK2 in the regulation of cell proliferation and survival. Cell. Mol. Life Sci. 66, 1817-1829 (2009).

22. Pinna, L. A. \& Allende, J. E. Protein kinase CK2 in health and disease: Protein kinase CK2: an ugly duckling in the kinome pond. Cell. Mol. Life Sci. 66 1795-1799 (2009)

23. Ruzzene, M. \& Pinna, L. A. Addiction to protein kinase CK2: a common denominator of diverse cancer cells? Biochim. Biophys. Acta 1804, 499-504 (2010).

24. Guerra, B. Protein kinase CK2 subunits are positive regulators of AKT kinase. Int. J. Oncol. 28, 685-693 (2006).

25. Di Maira, G. et al. Protein kinase CK2 phosphorylates and upregulates Akt/PKB. Cell Death Differ. 12, 668-677 (2005).

26. Hwang, D. W. et al. Autophagy induced by CX-4945, a casein kinase 2 inhibitor, enhances apoptosis in pancreatic cancer cell lines. Pancreas 46, 575-581 (2017).

27. So, K. S. et al. AKT/mTOR down-regulation by CX-4945, a CK2 inhibitor, promotes apoptosis in chemorefractory non-small cell lung cancer cells. Anticancer Res. 35, 1537-1542 (2015).

28. Bliesath, J. et al. Combined inhibition of EGFR and CK2 augments the attenuation of PI3K-Akt-mTOR signaling and the killing of cancer cells. Cancer Lett. 322, 113-118 (2012).

29. Ahmed, D. et al. Epigenetic and genetic features of 24 colon cancer cell lines. Oncogenesis 2, e71 (2013).

30. Scaffidi, P. et al. Release of chromatin protein HMGB1 by necrotic cells triggers inflammation. Nature 418, 191-195 (2002).

31. Cuyàs, E. et al. Cell cycle regulation by the nutrient-sensing mammalian target of rapamycin (mTOR) pathway. Methods Mol. Biol. 1170, 113-144 (2014).

32. Ramírez-Valle, F. et al. Mitotic raptor promotes mTORC1 activity, G2/M cell cycle progression, and internal ribosome entry site-mediated mRNA translation. Mol. Cell. Biol. 30, 3151-3164 (2010).

33. $Y u, C$. C. et al. RAD001 enhances the radiosensitivity of SCC4 oral cancer cells by inducing cell cycle arrest at the G2/M checkpoint. Anticancer Res. 34, 2927-2936 (2014).

34. Fan, B. et al. Inhibition of mTOR signaling protects photoreceptor cells against serum deprivation by reducing oxidative stress and inducing G2/M cell cycle arrest. Mol. Med. Rep. 13, 3771-3778 (2016).

35. Tapia, J. C. et al. Casein kinase 2 (CK2) increases survivin expression via enhanced beta-catenin-T cell factor/lymphoid enhancer binding factordependent transcription. Proc. Natl Acad. Sci. USA 103, 15079-15084 (2006).

36. Ponce, D. P. et al. Phosphorylation of AKT/PKB by CK2 is necessary for the AKTdependent up-regulation of $\beta$-catenin transcriptional activity. J. Cell. Physiol. 226, 1953-1959 (2011).

37. Ponce, D. P. et al. CK2 functionally interacts with AKT/PKB to promote the $\beta$ catenin-dependent expression of survivin and enhance cell survival. Mol. Cell. Biochem. 356, 127-132 (2011).

38. Francipane, M. G. \& Lagasse, E. mTOR pathway in colorectal cancer: an update. Oncotarget 5, 49-66 (2014)

39. Kim, K. W. et al. Autophagy for cancer therapy through inhibition of proapoptotic proteins and mammalian target of rapamycin signaling. J. Biol. Chem. 281, 36883-36890 (2006).
40. Recouvreux, M. V. \& Commisso, C. Macropinocytosis: A metabolic adaptation to nutrient stress in cancer. Front. Endocrinol. (Lausanne) 8, 261 (2017).

41. Overmeyer, J. H. et al. Active ras triggers death in glioblastoma cells through hyperstimulation of macropinocytosis. Mol. Cancer Res. 6, 965-977 (2008).

42. Overmeyer, J. H. et al. A chalcone-related small molecule that induces methuosis, a novel form of non-apoptotic cell death, in glioblastoma cells. Mol. Cancer 10, 69 (2011).

43. Lin, K. Y. et al. Overexpression of nuclear protein kinase CK2 Beta subunit and prognosis in human gastric carcinoma. Ann. Surg. Oncol. 17, 1695-1702 (2010).

44. Lin, K. Y. et al. Overexpression of nuclear protein kinase CK2 a catalytic subunit (CK2a) as a poor prognosticator in human colorectal cancer. PLOS ONE 6, e17193 (2011).

45. Ma, Z. et al. Increased expression of protein kinase CK2a correlates with poor patient prognosis in epithelial ovarian cancer. PLOS ONE 12, e0174037 (2017).

46. Niechi, I. et al. Colon cancer cell invasion is promoted by protein kinase CK2 through increase of endothelin- converting enzyme-1c protein stability. Oncotarget 6, 42749-42760 (2015).

47. Girardi, C. et al. Different persistence of the cellular effects promoted by protein kinase CK2 inhibitors CX-4945 and TDB. Biomed. Res. Int. 2015, 185736 (2015).

48. Zheng, $Y$. et al. Targeting protein kinase CK2 suppresses prosurvival signaling pathways and growth of glioblastoma. Clin. Cancer Res. 19, 6484-6494 (2013).

49. Gray, G. K. et al. Therapeutic CK2 inhibition attenuates diverse prosurvival signaling cascades and decreases cell viability in human breast cancer cells. Oncotarget 5, 6484-6496 (2014).

50. Polivka, J. \& Janku, F. Molecular targets for cancer therapy in the PI3K/AKT/ mTOR pathway. Pharmacol. Ther. 142, 164-175 (2014).

51. Brady, O. A. et al. Rags to riches: Amino acid sensing by the Rag GTPases in health and disease. Small GTPases 7, 197-206 (2016).

52. Olsen, B. B. et al. Downregulation of protein kinase CK2 induces autophagic cell death through modulation of the mTOR and MAPK signaling pathways in human glioblastoma cells. Int. J. Oncol. 41, 1967-1976 (2012).

53. Klionsky, D. J. et al. Guidelines for the use and interpretation of assays for monitoring autophagy (3rd edition. Autophagy 12, 1-222 (2016).

54. Matsumoto, G. et al. Serine 403 phosphorylation of p62/SQSTM1 regulates selective autophagic clearance of ubiquitinated proteins. Mol. Cell 44, 279-289 (2011).

55. Marquez, R. T. \& Xu, L. Bcl-2:Beclin 1 complex: multiple, mechanisms regulating autophagy/apoptosis toggle switch. Am. J. Cancer Res. 2, 214-221 (2012).

56. Maltese, W. A. \& Overmeyer, J. H. Methuosis: nonapoptotic cell death associated with vacuolization of macropinosome and endosome compartments. Am. J. Pathol. 184, 1630-1642 (2014).

57. Maltese, W. A. \& Overmeyer, J. H. Non-apoptotic cell death associated with perturbations of macropinocytosis. Front. Physiol. 6, 38 (2015).

58. Sander, P. et al. Vacquinol-1 inducible cell death in glioblastoma multiforme is counter regulated by TRPM7 activity induced by exogenous ATP. Oncotarget 8, 35124-35137 (2017).

59. Wang, P. N. et al. Downregulation of phosphorylated MKK4 is associated with a poor prognosis in colorectal cancer patients. Oncotarget 8, 34352-34361 (2017).

60. Kreutzer, J. N. et al. Enhancing chemosensitivity to gemcitabine via RNA interference targeting the catalytic subunits of protein kinase CK2 in human pancreatic cancer cells. BMC Cancer 10, 440 (2010).

61. Zhou, B. P. et al. HER-2/neu blocks tumor necrosis factor-induced apoptosis via the Akt/NF-kappaB pathway. J. Biol. Chem. 275, 8027-8031 (2000).

62. Yefi, R. et al. Protein kinase CK2 promotes cancer cell viability via up-regulation of cyclooxygenase-2 expression and enhanced prostaglandin E2 production. J. Cell. Biochem. 112, 3167-3175 (2011). 